\title{
Performance analyses of flowing chemical oxygen-iodine laser
}

\author{
Li-Min Hu and Zhi Gao
}

\begin{abstract}
A modified simplified rate-equation model that utilizes the Voigt profile function and another gain saturation model deduced from the kinetic equations are presented for performance analyses of a flowing chemical oxygen-iodine laser. Both models are adapted to both the condition of homogeneous broadening and that of inhomogeneous broadening being of importance and the condition of inhomogeneous broadening being predominant. Effects of temperature and iodine density on the output power and on variations of output power, optical intensity, and saturation intensity with flow distance are presented as well. There are differences between results of two models, but both qualitatively agree with known results. (C) 2003 Optical Society of America

OCIS codes: $140.1550,140.1340$.
\end{abstract}

\section{Introduction}

Performance analyses that predict the output power and the extraction efficiency of a chemical oxygeniodine laser (COIL) are of importance. To simplify the treatment of problems, a low-pressure limit model was used to calculate the output power and the extraction efficiency of a COIL under the condition of low pressure, 1,2 i.e., with inhomogeneous broadening being predominant. However, this approximation cannot predict the spectrum profile and cannot be utilized correctly in the cases of both homogeneous broadening and inhomogeneous broadening being of importance and nonzero frequency shift. Generally, the gas pressure in a laser cavity of flowing COIL is 1-10 Torr. $^{3}$ In this range, there are conditions of inhomogeneous broadening being predominant and both homogeneous and inhomogeneous broadening being of importance. Furthermore, the central frequency of the lasing spectroscopic line can vary in some cases; therefore a nonzero frequency shift will be produced.

Zagidullin et al. ${ }^{4}$ presented a model that takes into account both homogeneous and inhomogeneous broadening as well as velocity cross relaxation and a hyperfine relaxation process that can solve the above-

The authors are with the Institute of Mechanics, Chinese Academy of Sciences, Beijing 10080, China. H. Limin's e-mail address is musec@sina.com.

Received 22 August 2002.

0003-6935/03/336613-08\$15.00/0

(C) 2003 Optical Society of America described problems, but this model is quite complex and was not widely used. The most widely used theoretical model is still the rate-equation (RE) model, or its low-pressure limit in COIL. ${ }^{1-3}$ In this paper, a modified RE model that is adapted to both the condition of homogeneous broadening and inhomogeneous broadening being of importance and the condition of inhomogeneous broadening being predominant is presented.

However, as pointed out by some authors, when inhomogeneous broadening of low gas pressure is dominant, this kind of Voigt profile function models becomes inadequate. ${ }^{5,6}$ The gas pressure in the laser cavity of the COIL is generally several torrs, and thus the spectral line shape is inhomogeneously broadened; only part of the energy level atoms can interact directly with the monochromatic radiative field, whereas another part whose Doppler shift is large would not. However, the Voigt profile function model is not able to distinguish between these two parts of the particles and is not able to predict correctly the inhomogeneous broadening effects. To solve this, the semigas kinetics (SGK) model, ${ }^{5-9}$ which solved simultaneously the continuous medium equations for the macroscopic gas flow and the velocity distribution function equations for the lasing energy level particles, is used to analyze COIL and compare it with the results of $\mathrm{RE}$ model. The SGK model could predict exactly the anomalous gain saturation and the anomalous saturation line profile in $\mathrm{HF}$ chemical lasers, and the gain saturation theory of gas lasers (there is no macroscopic flow in it) and the $\mathrm{RE}$ model of gas-flow lasers were included in it as 
special cases. The performance calculation of supersonic hydrogen fluoride/deuterium fluoride (HF/DF) chemical lasers indicated that there is an obvious difference between the performance predictions of the SGK model and the RE model when both homogeneous broadening and inhomogeneous broadening are of importance, especially when inhomogeneous broadening is predominant.

The gas pressure range in a supersonic COIL cavity is nearly the same as that in the HF/DF chemical lasers. ${ }^{3,10}$ Therefore, in order to optimize the adjustable parameters of a supersonic COIL, it is necessary to deduce the SGK model for performance analyses of a supersonic COIL and compare performances predicted by the SGK model with those by the RE model. Apparently, in the development of supersonic HF/DF chemical lasers and supersonic COIL, it is quite important to ensure the optimization of adjustable parameters. For example, the output power was raised from a few watts of multimode limit to several thousand watts of near-diffraction limit in tens of years for flowing HF chemical lasers. ${ }^{10}$ There are also great disparities in the chemical efficiencies of supersonic COIL experiments. ${ }^{11}$ Herein a spectral line broadening (SLB) model is one of the key factors in explaining the disparities, and an appropriate SLB model can play an important role in optimizing the adjustable parameters. Thus it is important to examine and develop different SLB models in the COIL.

By use of both models, effects of temperature, pressure, iodine density, and frequency shift on the output power and the extraction efficiency of a COIL are presented as well.

\section{Gain Saturation Equation of the RE Model in Flowing Coil}

Similar to the research in Refs. 1 and 2, it is assumed that the primary flow containing excited state oxygen and the secondary flow containing iodine are premixed well before the laser cavity is entered, and the temperature and the pressure are constant everywhere in the cavity. The dissociation of iodine has occurred upstream of the laser cavity, and the deactivation in the cavity during the lasing process is negligible. It means that

$$
n_{1}+n_{2}=n,
$$

where $n_{1}$ and $n_{2}$ are the population of the excited state $I\left({ }^{2} P_{1 / 2}\right)$ and the ground state $I\left({ }^{2} P_{3 / 2}\right)$ (or the upper and lower laser level) of the atomic iodine, respectively; $n$ is the total particle number of the atomic iodine entering the laser cavity. The concentration of $\mathrm{O}_{2}\left({ }^{1} \Sigma\right)$ is assumed to be negligible. Because the flow velocity is quite high and the cavity length is relatively short, the flow transit time in the cavity is much shorter than the characteristic time for the quenching of the excited molecular oxygen $\mathrm{O}_{2}\left({ }^{1} \Delta\right)$; thus the effect of quenching is assumed to be negligible. Based on the above assumptions, the primary reaction in the cavity can be simplified as

$$
O_{2}\left({ }^{1} \Delta\right)+I \underset{k_{r}}{\stackrel{k_{f}}{\leftrightarrow}} O_{2}\left({ }^{3} \Sigma\right)+I^{*},
$$

where $k_{f}$ and $k_{r}$ are the forward and reverse rate constant, respectively; $k_{f}=2.3 \times 10^{-8} / T$; the temperature-dependent equilibrium constant $k_{\mathrm{eq}}=$ $k_{f} / k_{r}=0.75 \exp (401.4 / T) ; T$ is the flow temperature; and $I^{*}$ and $I$ denote the upper and lower laser levels of atomic iodine, respectively.

The gain expression is 7,10

$$
g=\sigma \varphi\left(\nu, v_{0}\right)\left(n_{2}-\alpha n_{1}\right),
$$

where $g$ is the gain, $\alpha$ is a constant related to level degeneracy for the COIL $\alpha \approx 1 / 2, \sigma$ is the pressuredependent stimulated emission cross section in square centimeters, $v$ and $v_{0}$ are the laser radiation frequency and the central frequency of the line profile, respectively, $\varphi$ is the Voigt profile function ${ }^{7,10}$

$$
\varphi\left(v, v_{0}\right)=\frac{\eta^{2}}{\sqrt{\pi}} \int_{-\infty}^{+\infty} \frac{\exp \left(-t^{2}\right)}{\eta^{2}+(\xi-t)^{2}} \mathrm{~d} t,
$$

where $\eta$ is the broadening parameter, $\eta=\left(\Delta v_{N} / \Delta v_{D}\right)$ $\sqrt{\ln 2}, \xi$ is the frequency-shift parameter, $\xi=[2(v-$ $\left.\left.v_{0}\right) / \Delta v_{D}\right] \sqrt{\ln 2}$, and $\Delta v_{N}$ and $\Delta v_{D}$ are the whole widths at half-height for the line profile of the Lorentz broadening and the Doppler broadening, respectively.

From Eqs. (1) and (3), it can be deduced that

$$
\begin{aligned}
& n_{1}=(n-g / \sigma \varphi) /(1+\alpha), \\
& n_{2}=(\alpha n+g / \sigma \varphi) /(1+\alpha) .
\end{aligned}
$$

Furthermore, the rate equations denoting the population variations of the upper and lower laser levels are

$$
\begin{aligned}
u \partial n_{2} / \partial x= & r n_{1}-k_{p} n_{2}-\sigma \varphi\left(\nu, v_{0}\right)\left(n_{2}-\alpha n_{1}\right) I /(h v) \\
u \partial n_{1} / \partial x= & -r n_{1}+k_{p} n_{2}+\sigma \varphi\left(\nu, v_{0}\right) \\
& \times\left(n_{2}-\alpha n_{1}\right) I /(h v)
\end{aligned}
$$

where $k_{p}$ and $r$ are the collision deactivation rate and the pumping rate of the upper laser level, respectively, and $u$ is the flow velocity in the streamwise direction. $I$ is the optical intensity, and $h v$ is the energy of a COIL photon. In addition,

$$
r=k_{f} n_{\Delta} \text { and } k_{p}=k_{r} n_{\Sigma},
$$

where $n_{\Delta}$ and $n_{\Sigma}$ are population of $\mathrm{O}_{2}\left({ }^{1} \Delta\right)$ and $\mathrm{O}_{2}\left({ }^{3} \Sigma\right)$, respectively.

From Eqs. (3), (5), and (6) it can be deduced that

$$
\begin{aligned}
u \frac{\partial g}{\partial x}+\left[\left(r+k_{p}\right)+(1+\alpha)\right. & \left.\frac{\sigma \varphi\left(v, v_{0}\right) I}{h v}\right] g \\
& =\left(r-\alpha k_{p}\right) \sigma \varphi\left(\nu, v_{0}\right) n .
\end{aligned}
$$


For the Fabry-Perot resonator, with the assumptions that the gain is equal to the loss in the laser cavity and the distributed loss and aperture loss are independent upon the distance $x$ in the streamwise direction (the so-called constant gain approximation), $, 7,10,12$ it can be deduced that

$$
g=g_{\text {th }}, \frac{\mathrm{d} g_{\text {th }}}{\mathrm{d} x}=0 .
$$

In the case of laser radiation frequency coinciding with the central frequency of the line profile, i.e., the frequency-shift parameter $\xi=0$, it can be deduced from Eqs. (7) and (8) that

$$
g=K \sigma n \eta \sqrt{\pi} \frac{\operatorname{erfc} \eta \exp \eta^{2}}{1+\bar{I}_{\nu} \eta \sqrt{\pi} \operatorname{erfc} \eta \exp \eta^{2}},
$$

where $K=\left(k_{f} n_{\Delta}-\alpha k_{r} n_{\Sigma}\right) /\left(k_{f} n_{\Delta}+k_{r} n_{\Sigma}\right)$ is a parameter that is introduced for writing convenience, $K \sigma n \eta \sqrt{\pi}=g_{0}$ is the small-signal gain, $\bar{I}_{v}$ is the dimensionless optical intensity, $\bar{I}_{v}=I / I_{s}$, and the saturated optical intensity $I_{s}=2 h v k_{r}\left[O_{2}\right]\left[\left(K_{e}-1\right) Y+\right.$ $1] /(3 \sigma)$ (where $Y$ is the yield of the molecular oxygen in the singlet delta state $\mathrm{O}_{2}\left({ }^{1} \Delta\right)$ and is defined as a fraction of total oxygen population, $Y=n_{\Delta} /\left[O_{2}\right]$, $\left[\mathrm{O}_{2}\right]=n_{\Delta}+n_{\Sigma}$ is the total population of the molecular oxygen).

When $\eta \gg 1$, i.e., the homogeneous broadening being predominant (high pressure limit), expansion of the error function in Eq. (9) gives approximately

$$
g=K \sigma n /\left(1+I / I_{s}\right) .
$$

When $\eta \ll 1$, i.e., the inhomogeneous broadening being predominant (low pressure limit), Eq. (9) gives approximately

$$
g=K \sigma n \eta \sqrt{\pi} /\left(1+I / I_{s} \eta \sqrt{\pi}\right) .
$$

\section{Comparison with SGK Model}

RE models are widely used in COIL. ${ }^{1-3}$ From the deduction and analyses above, it can be concluded that RE model can consider conditions of inhomogeneous broadening being predominant and both homogeneous and inhomogeneous broadening being of importance, and it can predict effects of pressure and frequency shift on the performance of COIL. However the RE model cannot consider frequency characteristics of the spectral line profile and cannot explain the anomalous gain saturation in $\mathrm{HF} / \mathrm{DF}$ chemical laser experiments. An SGK model ${ }^{6-10}$ can solve simultaneously the continuous medium equations for the macroscopic gas flow and the velocity distribution function equations for the lasing energy level particles. Thus the SGK model could predict exactly the anomalous gain saturation and anomalous saturation line profile in HF chemical lasers, and the gain saturation theory of gas lasers (there is no macroscopic flow in it), and the RE model of gas flow lasers were included in it as special cases. The performance calculation of supersonic $\mathrm{HF} / \mathrm{DF}$ chemical lasers indicated that there is an obvious difference between the performance predictions of the SGK model and the RE model when both homogeneous broadening and inhomogeneous broadening are of importance, especially when inhomogeneous broadening is predominant, i.e., the RE model's expression equation of the high-pressure limit is in consistent with that in chemical laser ${ }^{13}$ and the SKG model, ${ }^{6-9}$ but its low-pressure limit model is quite different from that of chemical laser and the SGK models, as both the low- and high-pressure limit of SGK model are in consistent with those of chemical laser. For these reasons, it should be useful to compare both $\mathrm{RE}$ and SGK models to find out the difference.

In the SGK model, the gas kinetic equations that describe the translational velocity distributions of particles in the upper and lower iodine energy levels are presented, and a method for solving these equations is developed. ${ }^{6-9}$ The gain saturation equation is obtained as

$$
g=K \sigma n \frac{\eta^{2}(1+\bar{I})}{\sqrt{\pi}} \int_{-\infty}^{+\infty} \frac{e^{-t^{2}}}{\eta^{2}(1+\bar{I})+(\xi-t)^{2}} \mathrm{~d} t .
$$

In the case of the frequency-shift parameter $\xi=0$, it can be deduced from Eq. (12) that

$$
g=K \sigma n \frac{\eta \sqrt{\pi}}{\sqrt{1+\bar{I}}} \exp \left[(1+\bar{I}) \eta^{2}\right] \operatorname{erfc}(\eta \sqrt{1+\bar{I}}) .
$$

Here $\bar{I}$ is the dimensionless optical intensity of SGK model, $\bar{I}=I / I_{S}$.

When $\eta \gg 1$, i.e., the homogeneous broadening being predominant (high-pressure limit), expanding the error function in Eq. (13), an equation that is the same as Eq. (10) of the RE model can be obtained.

When $\eta \ll 1$, i.e., the inhomogeneous broadening being predominant (low-pressure limit), Eq. (13) gives

$$
g=K \sigma n_{0} \frac{\eta \sqrt{\pi}}{\sqrt{1+I / I_{S}}} .
$$

It differs greatly from Eq. (11) of the RE model under the condition of low gas pressure.

Both approximate expressions of the SGK model at high and low gas pressure are inconsistent with the gain saturation expressions of the gas laser. ${ }^{13}$

\section{Calculation of Output Power and Extraction Efficiency}

The output power $P$ can be expressed as ${ }^{1,2}$

$$
\begin{aligned}
P & =P_{\text {av }} \eta_{\text {ext }}, \\
\eta_{\text {ext }} & =\eta_{\text {extm }} \eta_{\text {extr }},
\end{aligned}
$$

where $P_{\text {av }}$ is the maximum available power; $\eta_{\text {extr }}$ is the extraction efficiency of the resonator, which is dependent upon the type of optical resonator, the mirror absorbability, and the reflectivity and is approximately independent upon the kind and the flow rate of the gas medium and which is a known param- 
eter; $\eta_{\text {extm }}$ is the medium extraction efficiency for extracting power from the gain medium, which describes the availability of the laser medium; $\eta_{\text {ext }}$ is the extraction efficiency or efficiency of the whole system, including the optical resonator and gain medium. For a COIL, 1,2 it can be deduced that

$$
\begin{aligned}
P_{\mathrm{av}} & =h v\left(Y_{o}-Y_{\mathrm{th}}\right) Q N_{A}, \\
\eta_{\text {extm }} & =\frac{Y_{0}-Y_{e}}{Y_{0}-Y_{\mathrm{th}}},
\end{aligned}
$$

where $Y_{0}$ and $Y_{e}$ denote yield at the entrance and the exit of the laser cavity, respectively; $Y_{\mathrm{th}}\left[=1 /\left(2 k_{\mathrm{eq}}+\right.\right.$ $1)$ ] is the threshold yield; $Q$ is the molar flow rate of oxygen (in moles per second); and $N_{A}$ is the Avogadro number. In the following, the medium extraction efficiency is deduced from the expression of gain.

As noted above, it is assumed that a quenching of the excited iodine in the cavity is negligible and the molecular iodine has been dissociated completely before entering the laser cavity, so total energy stored in the singlet oxygen can be transformed into the laser energy completely: ${ }^{1,2}$

$$
u \frac{\mathrm{d} n_{\Delta}}{\mathrm{d} x}=-\frac{g I}{h v} .
$$

For a Fabry-Perot resonator and based on the constant-gain approximation, ${ }^{7,10,12} \mathrm{~g}$ is equal to the threshold gain and is a constant in the streamwise direction $x$.

In the case of low gas pressure, i.e., inhomogeneous broadening, it is deduced from Eqs. (11) and (19) with the boundary condition at the cavity entrance $\left.Y\right|_{x=0}=$ $Y_{0}$ that

$$
\left.Y=Y_{0}-\frac{q}{p} \exp (-p x)\right]+\frac{q}{p},
$$

where $p$ and $q$ are two parameters introduced for writing convenience and

$$
\begin{aligned}
& p=\frac{2 k_{r}}{3 \eta \sqrt{\pi} \sigma u}\left[\frac{\eta \sqrt{\pi} \sigma n}{2}\left(2 K_{e}+1\right)-\left(K_{e}-1\right) g\right] Y, \\
& q=\frac{2 k_{r}}{3 \eta \sqrt{\pi} \sigma u}\left(\frac{\eta \sqrt{\pi} \sigma n}{2}+g\right) .
\end{aligned}
$$

When expression of the Doppler stimulated emission cross section $\sigma_{D}(=\sigma \eta \sqrt{\pi})$ (Ref. 3) is introduced into Eq. (20), the obtained distribution of yield $Y$ is completely the same as that in Ref. 1.

In the cases of both homogeneous broadening and inhomogeneous broadening being of importance and/or the nonzero frequency shift, two approximation expressions of the homogeneous broadening and the inhomogeneous broadening are not adapted, and a set of equations composed with Eqs. (19) and (9) [or (13)] and the entrance boundary condition $\left.Y\right|_{x=0}=Y_{0}$ must be solved numerically to obtain the distribution of yield $Y$ and further the extraction efficiency and the output power of the laser device.

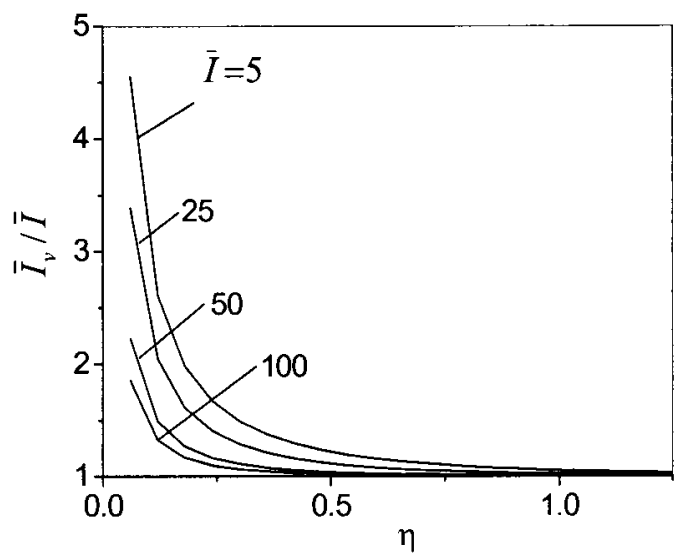

Fig. 1. Relations between dimensionless intensity $\bar{I}$ of Eq. (7) and $\bar{I}_{\nu}$ of Eq. (12).

\section{Results and Discussion}

In the following, a part of results obtained from the present modified RE model are compared with the experimental observations in the RotoCOIL. ${ }^{11,14}$ The expression of the resonator extraction efficiency is taken from Refs. 2 and 11:

$$
\begin{aligned}
\eta_{\text {extr }}= & \left(1-R_{\text {out }}-S_{\text {out }}\right) /\left[\left(1-R_{\text {out }}-S_{\text {out }}\right)(1+\delta)\right. \\
& \left.+S_{\text {out }}+\left(R_{\text {out }} / R_{\max }\right)^{1 / 2}\left(1-R_{\text {max }}\right)\right]
\end{aligned}
$$

where $R_{\text {out }}$ (variable) is the reflectivity of the outcoupling mirror, $R_{\max }(=0.995)$ is the reflectivity of the highly reflective mirror, $S_{\text {out }}=0.004$ is the scattering coefficient of the outcoupling mirror, and $\delta=0.13$ is the diffraction loss fraction. The threshold gain is dependent upon $R_{\text {out }}$, and $P_{\text {av }}$ is expressed by Eq. (14). The flow conditions within the laser cavity are deduced by analysis and calculation of the RotoCOIL experiment 11,14 : The flow temperature $T$ is $167 \mathrm{~K}$, the entrance pressure $P$ is approximately 4 torr, the small signal gain $g_{0}=0.0068 \mathrm{~cm}^{-1}$, the cavity yield $Y_{0}=0.42$, the molar flow rate of oxygen $Q=1.395$ $\mathrm{mol} / \mathrm{sec}$, the dilution ratio is approximately $\mathrm{He}: \mathrm{O}_{2}=$ $4: 1$, the flow ratio of $\mathrm{I}_{2}$ and $\mathrm{O}_{2}$ is $\mathrm{I}_{2} / \mathrm{O}_{2}=0.0158$, and the flow velocity $u=84100 \mathrm{~cm} / \mathrm{s}$ (Ref. 1 ). In the simulation computation of the experiment, the broadening parameter $\eta=0.08$ is taken in this paper. The calculation herein is based on the RotoCOIL experiment, that is, chosen parameters and their related variables are determined on the base of experimental data, and other parameters are directly taken to be the experimental data.

The correlations between $\bar{I}$ and $\bar{I}_{v}$ are shown in Fig. 1. $\bar{I}$ and $\bar{I}_{v}$ are nearly the same when $\eta$ is not small; for example, when $\eta=0.75$ the differences are nearly negligible, and the smaller the value of $\eta$, the larger the difference between $\bar{I}$ and $\bar{I}_{v}$.

Figure 2 shows a comparison of the result by using the present modified RE model, and the SGK model with the RotoCOIL experimental data and results of paper. ${ }^{1}$ It is shown that the agreement is quite good. The theoretical result of the RE model is quite closed to that in Ref. 1; the result of the SGK model 


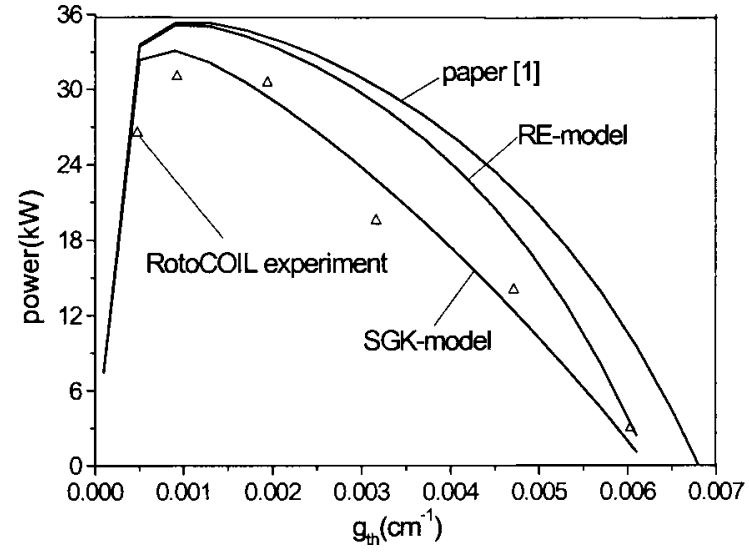

Fig. 2. Comparison of theoretical powers with RotoCOIL experimental data.

is less but is closer to experiment data. Strictly speaking, the model of Ref. 1 is only adapted to the case of lower pressure. However, the Voigt profile is used in the present RE model; thereby the pressure can be extended to a broader range and the present modified model can be used to deal with the problem of both homogeneous broadening and inhomogeneous broadening being of importance.

Figure 3 shows a comparison of the extraction efficiency of the RE model for different broadening parameter $\eta$ with the result of Ref. 1 ; here, constant flow rates of the lasing medium and the energy-stored medium are assumed. The result of Ref. 1 corresponds to the case when $\eta=0$. The difference of the $\mathrm{RE}$ model result with that of Ref. 1 is quite small when $\eta$ is small; as $\eta$ gets larger, the difference of the two models gradually becomes larger. As $\eta$ increases, the threshold gain at zero power crossing point (denoted by $g_{\text {tho }}$; there exist laser oscillation and output power when $g_{\text {th }}<g_{\text {th0 }}$ ) decreases. Thus, when $\eta \geq 0.1$ (correspondingly, pressure $p \geq 4$ torr), it is necessary to consider effects of the homogeneous broadening and the inhomogeneous broadening simultaneously.

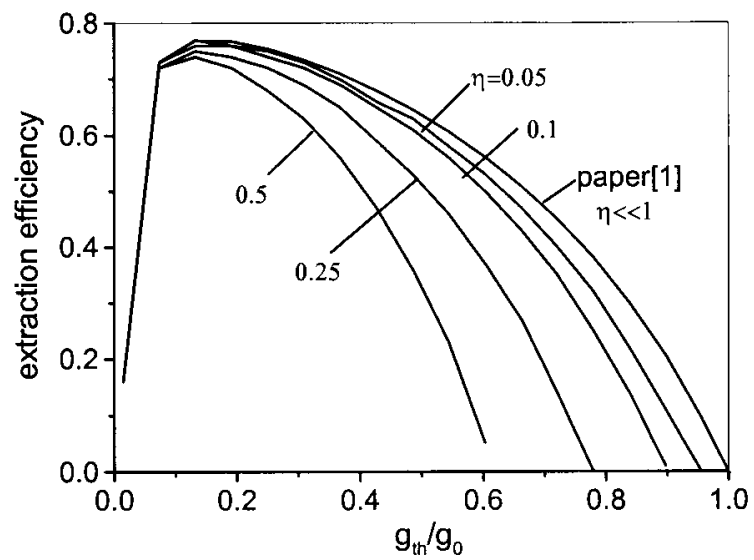

Fig. 3. Comparison of efficiencies of Ref. 1 and RE model for different $\eta$.

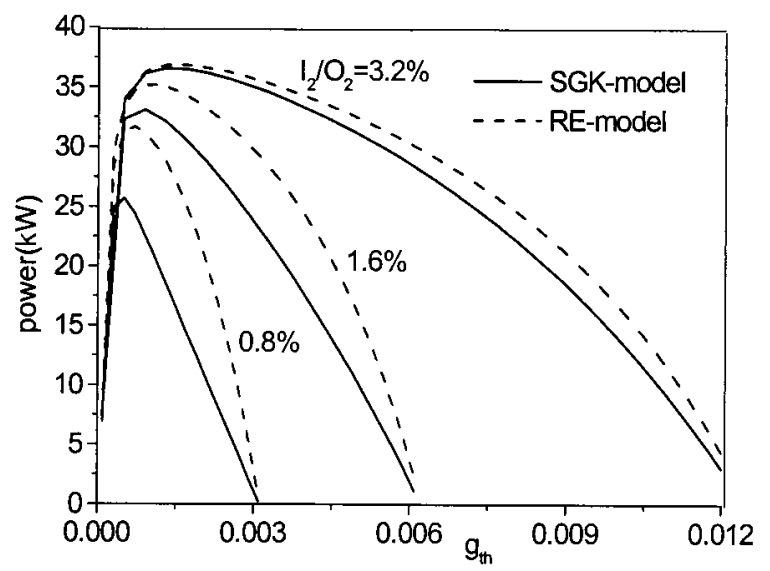

Fig. 4. Variation of power with $g_{\text {th }}$ for different $\mathrm{I}_{2} / \mathrm{O}_{2}$ values.

Figure 4 shows variations of the output powers with $g_{\text {th }}$ for different flow ratio $\mathrm{I}_{2} / \mathrm{O}_{2}$ by use of the $\mathrm{RE}$ and SGK models (here the oxygen flux is constant and only the iodine flux varies; the small-signal gain $g_{0}$ varies in proportion to the variation of $\mathrm{I}_{2}$ flux). The output power increases with the increment of iodine flux. When $\mathrm{I}_{2}$ flux is large, the difference between results of both models is small, but results of $\mathrm{RE}$ model become much greater than that of the SGK model results when the $I_{2}$ flux becomes smaller, therefore increasing the atomic iodine concentration is favorable for an increasing of the output power.

The lasing frequency does not always coincide with the central frequency of the laser spectroscopic line, and it is possible for a certain frequency shift to exist. ${ }^{15}$ Figure 5 shows variations of the output power with $g_{\text {th }}$ for different frequency-shift parameter $\xi$ and different models. It is shown that the output power decreases as the frequency shift increases. When the frequency-shift parameter $\xi$ reaches 1 , the output power decreases rapidly as the threshold gain increases. Results of the $\mathrm{RE}$ model are distinctly greater than that of the SGK model for same frequency-shift value.

Figure 6 shows variations of the optimum output power (the output power at the optimum threshold

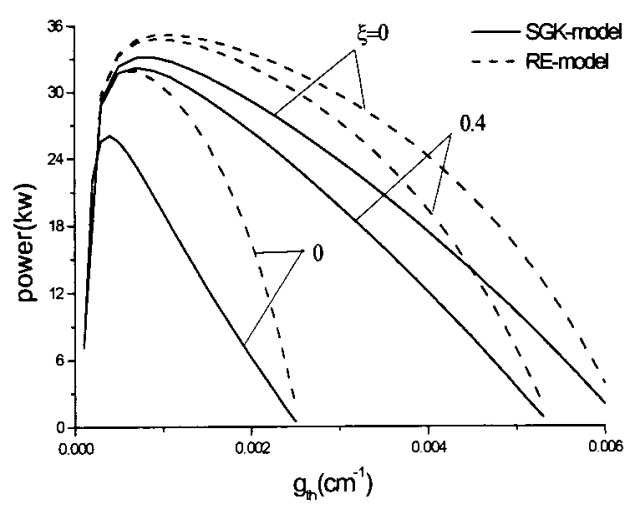

Fig. 5. Variation of power with $g_{\text {th }}$ for different $\xi$. 


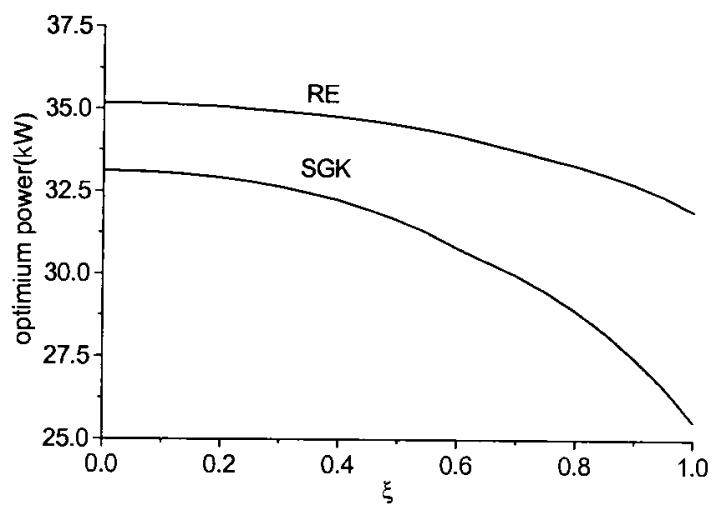

(a)

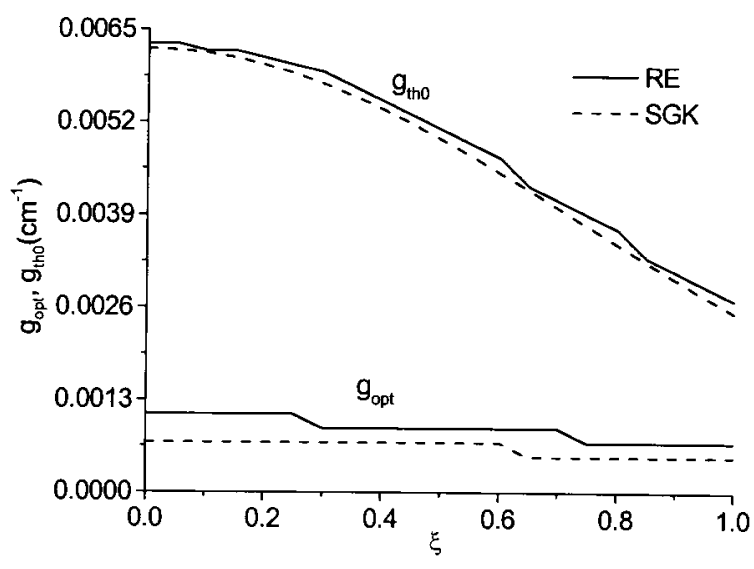

(b)

Fig. 6. Variations of (a) the optimum power and (b) the optimal threshold gain $g_{\text {opt }}$ and zero-power crossing point $g_{\text {tho }}$ with $\xi$ in $\mathrm{RE}$ and SGK models.

gain), the optimum threshold gain $g_{\text {opt }}$, and the threshold gain at zero power crossing point $g_{\text {tho }}$ with the frequency-shift parameter $\xi$ for the $\mathrm{RE}$ and the SGK models. As the frequency shift increases, the optimum threshold gain $g_{\text {opt }}$ decreases slightly, and the threshold gain at zero power crossing point $g_{\text {tho }}$ decreases gradually. The differences of $g_{\text {opt }}$ and $g_{\text {tho }}$ between both models are small, and the varying trends are similar, but the optimal powers of the two models differ substantially, as shown in Fig. 6(a).

Figure 7 shows variations of the output power with the threshold gain $g_{\text {th }}$ for different temperature $T$ of both the RE and the SGK models. Since a decrease of temperature is favorable for exciting the atomic iodine from the lower energy level to the excited state, the small-signal gain increases. Furthermore, a decrease of temperature results in an increase of the maximum available power $P_{\mathrm{av}}$. Therefore when the temperature is lower, the output power is higher. In addition, it is also shown that as temperature decreases, the threshold gain at zero-power crossing point $g_{\text {tho }}$ increases and the range of threshold gain having higher output power is enlarged as well. Both models show the same varying trend, but the

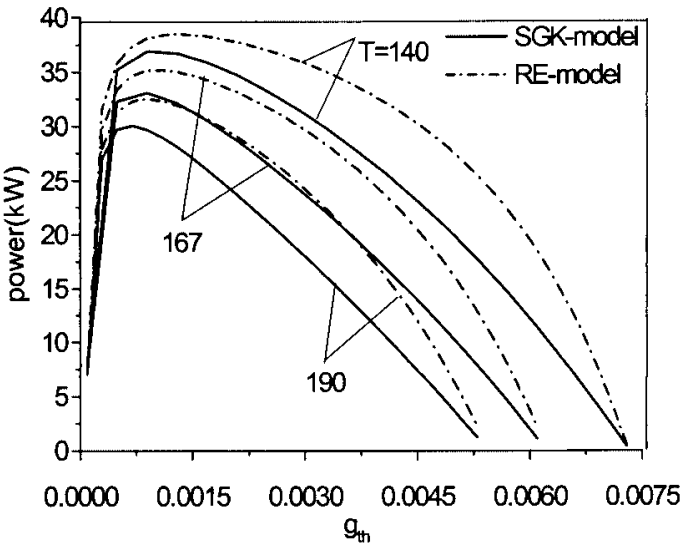

Fig. 7. Comparison of powers with use of the SGK and RE models.

powers of the RE model are greater than that of the SGK model. Thereby a decrease of temperature by means of increasing the expansion Mach number is an important provision for improving the laser device performances.

Figure 8 shows variations of the output power with $g_{\text {th }}$ for different yield values of the excited molecular oxygen $Y_{0}$ in the RE and SGK models. Both models show that as the yield of $\mathrm{O}_{2}\left({ }^{1} \Delta\right)$ increases, the threshold gain at zero-power crossing point $g_{\text {tho }}$ increases slightly; the ranges of threshold gain existing output power, and having a higher output power are enlarged. Thus an increase of the yield of $\mathrm{O}_{2}\left({ }^{1} \Delta\right)$ can make a great contribution to increase the output power.

Figure 9 shows respectively variations of the output power, the saturation degree $S$ (defined as 1 $g_{\text {th }} / g_{00}$ when strong light signal exists $\left.{ }^{13}\right)$, the optical intensity $I$ and the $\mathrm{O}_{2}\left({ }^{1} \Delta\right)$ yield $Y$ with the distance downstream by use of both the RE and the SGK models, together with equations in Ref. 1. Here $g_{\text {th }}=0.0025 \mathrm{~cm}^{-1}$, and other values are taken as experimental data.

Figure 9(a) shows that the output powers increase quicker near the entrance than downstream in all models. The output power of the SKG model increases slowly along the mirror length, but it is obvious that the power extraction of the $\mathrm{RE}$ model

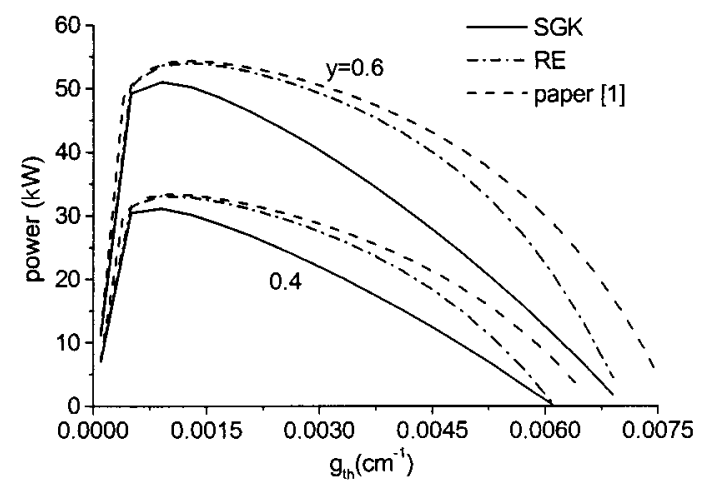

Fig. 8. Variations of powers with $g_{\text {th }}$ for different $Y_{0}$. 


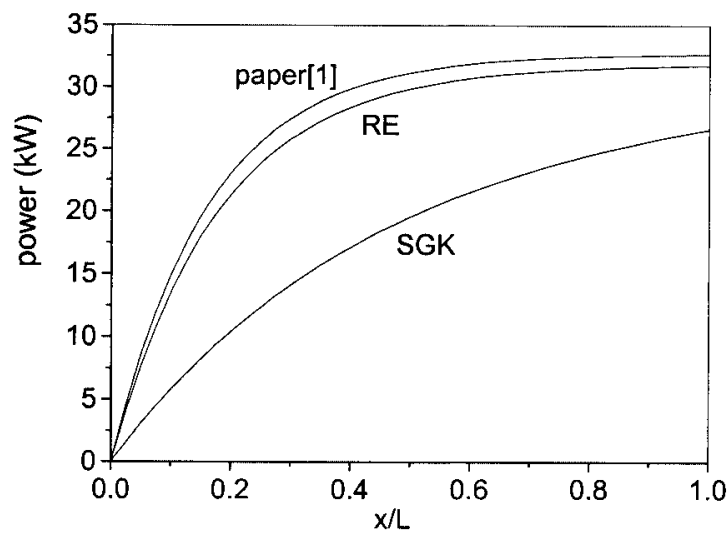

(a)

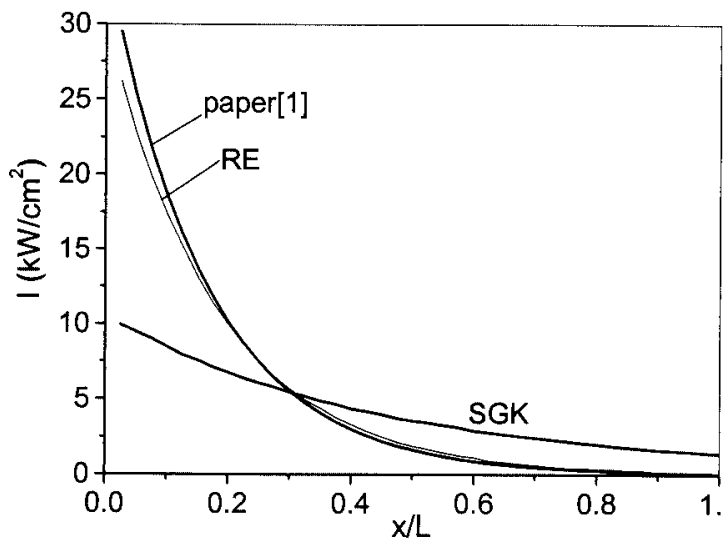

(c)

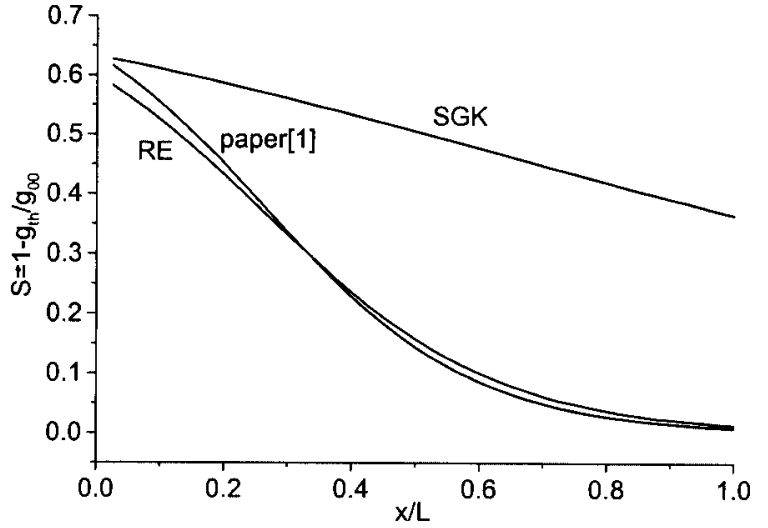

(b)

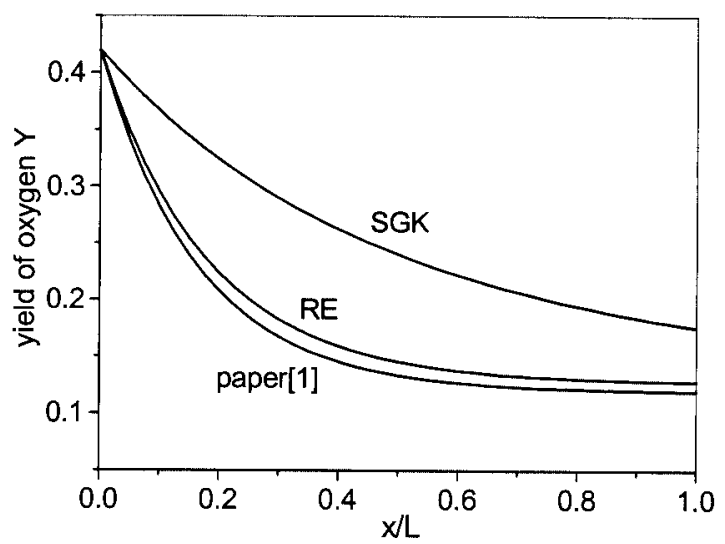

(d)

Fig. 9. Variations of (a) the output power, (b) the gain saturation degree, (c) the optical intensity, and (d) the excited oxygen yield with $x / L$ when $g_{\text {th }}=0.0025 \mathrm{~cm}^{-1}$.

and the Ref. 1 results are much quicker and is nearly completed in a shorter mirror length, i.e., the mirror length is unnecessarily longer according to the RE model and the Ref. 1 theory. Figure 9(b) shows that the saturation degree of the RE model and the results of Ref. 1 are quite close along the $x$ direction, but the SGK model's saturation degree is greater than those of the other two and decreases much more slowly.

Figure 9(c) shows that the optical intensity of the $\mathrm{RE}$ model is much greater than that of the SGK model near the entrance and smaller at the exit, and it decreases more rapidly along the streamwise direction. As to the variations of the oxygen yield in Fig. 9(d), the SGK model predicts a slower decrease, and more residual energy of excited oxygen remains in the exhaust gas that flows out of the laser cavity.

It can be concluded that the RE model and Ref. 1 theory predict a more rapid power extraction process and a higher output power than the SGK model does. Moreover, the power extraction length of the $\mathrm{RE}$ model is shorter than that of the SGK model. These results are important and useful to the design of a COIL laser cavity.

\section{Conclusion and Review of Results}

A modified RE model is presented for performance analyses of a flowing COIL and compared with a new SGK model. Both models can be utilized to a larger pressure range wherein both the condition of homogeneous broadening and inhomogeneous broadening being of importance and the condition of inhomogeneous broadening being predominant exist. The results agree well with experimental data and previous results. By simulating an experiment with a premixed simplified flow model, the modified RE model and the SGK model considered the effects of pressure on the power and extraction efficiency of a COIL that have not previously been performed in the COIL. From the formula deduction and calculation results, both models show that an increase of pressure, temperature, and frequency shift will reduce the output power and the extraction efficiency of COIL, whereas an increase of the atomic iodine density can increase the output power and the extraction efficiency of the COIL. These results about temperature, iodine density, distribution of optical intensity, and oxygen yield along the flow direction agree well with the previous theory ${ }^{1}$ and show that both the $\mathrm{RE}$ and the 
SGK model can be utilized to the performance analysis of COIL efficiently.

However, as pointed out in the introduction, the RE model is not able to predict correctly the inhomogeneous broadening effects, for it must consider finite translational relaxation rates, whereas SGK model solved this difficulty by seeking a double-parameter perturbation solution of the conservation equation of the velocity distribution function of lasing particles, and more physical contents are included in SGK model. The differences between the computed results of the RE and SGK models are useful to the optimization of adjustable parameters in flowing COILs.

This research is supported by the National Foundation of Natural Sciences of China (Project No. 10032050, 10272106).

\section{References}

1. G. D. Hager, C. A. Helms, K. A. Truesdell, D. Plummer, J. Erkkila, and P. Crowell, "A simplified analytic model for gain saturation and power extraction in the flowing chemical oxygen-iodine laser," IEEE J. Quantum Electron. 32, 15251536 (1996).

2. B. D. Barmashenko and S. Rosenwaks, "Analysis of the optical extraction efficiency in gas-flow lasers with different types of resonator," Appl. Opt. 35, 7091-7101 (1996).

3. Q. Zhuang, F. T. Sang, and D. Z. Zhou, Short-Wavelength Chemical Lasers (Press of National Defense Industry, Beijing, China, 1997), in Chinese.

4. M. V. Zagidullin, V. I. Igoshin, and N. L. Kupriyanov, "Kinetics of saturation of the active medium of an oxygen-iodine laser," Sov. J. Quantum Electron. 14, 930-936 (1984).

5. H. Mirels, "Effects of translational and rotational nonequilib- rium on cw chemical laser performance," Appl. Opt. 27, 89-95 (1988).

6. Z. Gao, "Collisional and inhomogeneous broadening effects in gas-flow and chemical lasers: the theoretical models," Acta Phys. Sin. 30, 1591-1602 (1981), in Chinese.

7. Z. Gao and E. Xuequan, "Kinetics model of flowing chemical lasers," Sci. China A 31, 46-57 (1982), in Chinese.

8. Y. Hai-Xing and Z. Gao, "Laser-gas flow medium interactions and their numerical simulations," in Seventh International Symposium on Gas Flow and Chemical Lasers Proc. SPIE 1031, 532-544 (1989).

9. G. Zhi and H. Limin, "Effects of spectral line broadened model on performance of flowing chemical oxygen-iodine laser," Chin. Phys. Lett. 19, 1628-1631 (2002).

10. R. W. F. Gross and J. F. Bott, Handbook of Chemical Lasers (Wiley, New York, 1976), p. 501.

11. J. F. Hon, D. N. Plummer, P. G. Crowell, J. Erkkila, G. D. Hager, C. A. Helms, and K. A. Truesdell, "A heuristic method for evaluating COIL performance," presented at the 25th America Institute of Aeronautics and Astronautics Plasmadynamics and Laser Conference, Colorado Springs, Colo., 20-23 June 1994, paper 94-2422.

12. A. Elior, B. D. Barmashenko, E. Lebiush, and S. Rosenwaks, "Experiment and modeling of a small scale, supersonic oxygeniodine laser," Appl. Phys. B 61, 37-47 (1995).

13. Y. Chen and J. Wang, Principles of Lasers (Zhejiang U. Press, Hangzhou, China, 1998) pp. 299-300 (in Chinese).

14. K. A. Truesdell, C. A. Helms, and G. D. Hager, "COIL development in the USA," presented at the American Institute of Aeronautics and Astronautics 25th Plasmadynamics and Laser Conference, Colorado Springs, Colo., 20-23 June 1994, paper 94-2421.

15. D. A. Copeland and A. H. Bauer, "Optical saturation and extraction from the chemical oxygen-iodine laser medium," IEEE J. Quantum Electron. 29, 2525-2539 (1993). 